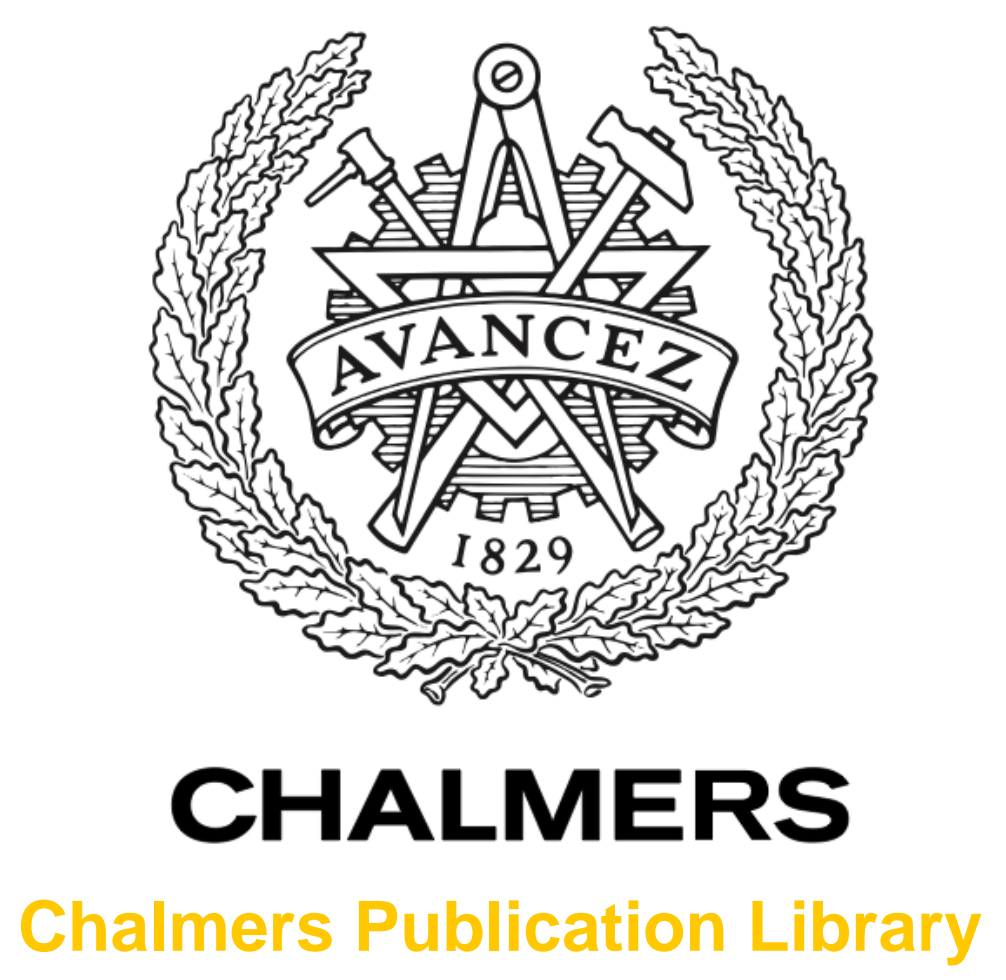

\title{
Model-Based Threat Assessment for Lane Guidance Systems
}

This document has been downloaded from Chalmers Publication Library (CPL). It is the author's version of a work that was accepted for publication in:

\author{
American Control Conference, San Francisco, CA, USA, June 29-July 01, 2011 (ISSN: \\ 07431619) \\ Citation for the published paper: \\ Ali, M. ; Falcone, P. ; Sjöberg, J. (2011) "Model-Based Threat Assessment for Lane \\ Guidance Systems". American Control Conference, San Francisco, CA, USA, June 29-July \\ 01, 2011 pp. 4586-4591.
}

Downloaded from: http://publications.lib.chalmers.se/publication/136612

Notice: Changes introduced as a result of publishing processes such as copy-editing and formatting may not be reflected in this document. For a definitive version of this work, please refer to the published source. Please note that access to the published version might require a subscription. 


\title{
Model-Based Threat Assessment for Lane Guidance Systems
}

\author{
Mohammad Ali, Paolo Falcone and Jonas Sjöberg
}

\begin{abstract}
This paper considers a threat assessment problem in a lane guidance application for semi-autonomous vehicles. In particular, in order to issue an autonomous assisting intervention, we assess the vehicle's ability to safely travel along a path subject to limitations arising from the vehicle's dynamics and the driver's ability.

We first introduce a set of constraints describing "safe driving". For the specific lane guidance application considered in this paper, the constraints are set by the lane boundaries and the vehicle's stability limits. We then formulate the threat assessment problem as a constraints satisfaction problem over a finite time horizon, solved by resorting to reachabililty analysis and invariant set theory.

Validation with experimental data demonstrates the capability of the proposed threat assessment method of predicting vehicle instability or crossing of the lane boundaries.

Index Terms - Invariant Set Theory, Semi-Autonomous Vehicles, Threat Assessment, Decision Making, Active Safety.
\end{abstract}

\section{INTRODUCTION}

In this paper, we focus on semi-autonomous vehicles, i.e., human driven vehicles with autonomous driving capabilities. We consider lane guidance applications, where the driver has to be assisted in maintaining the vehicle within the lane boundaries, through assisting autonomous driving interventions. A challenging aspect of such problems is to determine when the driver needs assistance since an assisting autonomous driving intervention should be issued if and only if a risk of accident is detected, that the driver is not able to avoid. The formulation of transition conditions, between the different modes of a semi-autonomos vehicle, e.g., from a driver-controlled to a fully autonomous assisting mode, is not trivial.

In the threat assessment methods considered in this paper, we first introduce a set of constraints describing "safe" driving, i.e., the constraints satisfaction would result in an accident free driving. Moreover, we assume the road geometry is available over a future finite time horizon and exploit the vehicle modeling in order to calculate a "safe set". This is the set of vehicle states at current time $t$ for which a steering trajectory exists such that, over a finite time horizon, the vehicle state evolves to a given final set without violating constraints. Once the safe set is computed, a set membership test on the current vehicle state is performed. If the current state belongs to the safe set, a steering trajectory exists such that the vehicle can be driven over the considered future time horizon without exceeding its stability limits and leaving

P. Falcone and J. Sjöberg are with Chalmers University of Technology, Dept. of Signals and Systems , 41296 Göteborg, \{falcone,jonas.sjoberg\}@chalmers.se

M. Ali is with Volvo Car Corporation, Active Safety \& Chassis, 96410/PV4A, 40531 Göteborg, mali21@volvocars.com the lane. We remark that the safe set can be used in the autonomous driving mode to determine when the control of the vehicle can be released and given back to the driver. Moreover, the safe set can be used as a target set where the autonomous driving system should steer the vehicle state in order to let the driver recover the vehicle control and safely complete the driving task.

Similar ideas can be found in [1], [2], [3] in the automotive and aerospace fields, respectively. In [1], the authors propose a collision detection method in autonomous driving. In particular, a traffic scenario is considered where the path of an autonomous ego car has to be planned in order to avoid collisions with other traffic participants, whose future trajectory is unknown. A safe planned path for the ego car is required to not intersect the stochastic reachable sets computed for each traffic participant, i.e., the future set of positions possibly occupied by the other traffic participants. In [2], a safety analysis of an aircraft autoland system is developed based on the calculation of reachable sets. In the landing phase, the set of the aircraft configurations, evolving within a safe envelope to the set of acceptable states at touchdown, is calculated as a backward reachable set. The algorithm in [3] is used, computing the reachable sets of a nonlinear system based on a time-dependent Hamilton-Jacobi PDE.

We demonstrate the proposed threat assessment method in a roadway departure application, and validate it through experimental data. The paper is organized as follows. In Section II we overview the considered semi-autonomous driving architecture while in Section III, we provide basic definitions and results on reachability analysis and set invariance theory. In Section IV, we present the modeling used in Section V, where the threat assessment algorithm is presented. In Section VI, we validate the proposed algorithm and in Section VII we close the paper with final remarks.

\section{SYSTEM OVERVIEW}

We consider the accident avoidance architecture presented in [4] and sketched in Figure 1. The architecture is general and can be used in any accident avoidance or mitigation application. In Figure 1, we distinguish a threat assessment and an intervention layer. The two layers share information about the environment provided by the environment information block.

Based on measurements and estimation of the vehicle state, the threat assessment utilizes preview information in order to evaluate the risk of roadway departure or vehicle instability. As long as the threat assessment indicates a low threat level, the driver has full control of the vehicle. The type 


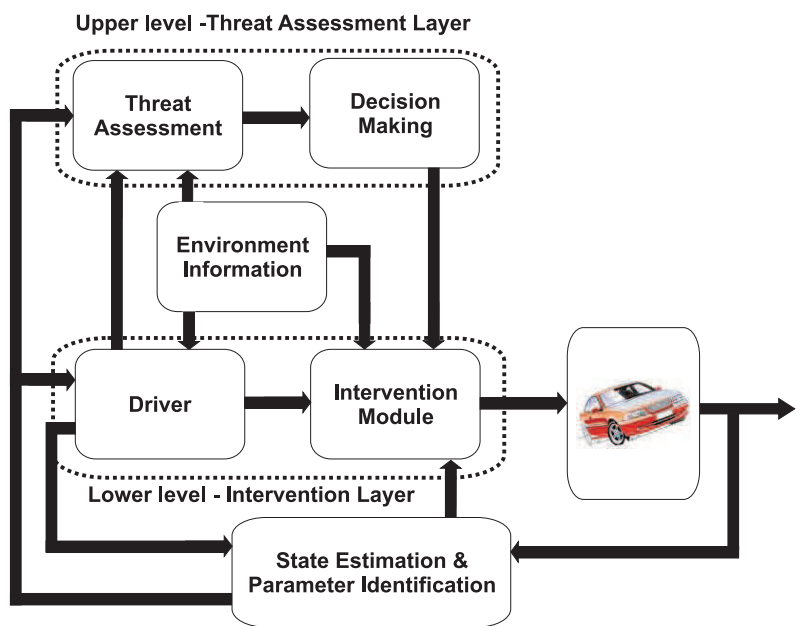

Fig. 1. Overview of the suggested accident prevention architecture.

of intervention issued, can then range from merely assisting the driver by slightly correcting his or her control action to completely excluding the driver in a completely autonomous mode. The output of the decision making module can thus be the choice of a lower level controller implemented in the intervention module. A mathematical formulation of such a decision making framework along with an example of a lower level controller that utilizes combined front steering and differential braking in order to follow a safe path is provided in [4]. In the rest of the paper, we focus on the threat assessment module of the architecture in Figure 1.

\section{BACKGROUND ON SET INVARIANCE THEORY AND REACHABILILTY ANALYSIS}

This section adopts the notation used in [5], [6], [7] and provides the basic definitions of invariant and reachable sets for constrained systems. A comprehensive survey of papers on set invariance theory can be found in [8]. We will denote the set of all real numbers and positive integers by $\mathbb{R}$ and $\mathbb{N}^{+}$, respectively.

Denote by $f$ the state update function of the discrete-time system with inputs

$$
x(t+1)=f(x(t), u(t), w(t)),
$$

where $x(t), u(t)$ and $w(t)$ denote the state, input and disturbance vectors, respectively. System (1) is subject to the constraints

$$
x(t) \in \mathcal{X}, u(t) \in \mathcal{U}, w(t) \in \mathcal{W} .
$$

The set of states which can be driven into the target set $\mathcal{S}$ in one time step is defined as

$$
\operatorname{Pre}_{f}(\mathcal{S}) \triangleq\{x \in \mathcal{X} \mid \exists u \in \mathcal{U} \text { s.t. } f(x, u) \in \mathcal{S}\}
$$

The following definitions are derived from [8], [9], [10], [11].

Definition 1 (Control Invariant Set): A set $\mathcal{C} \subseteq \mathcal{X}$ is said to be a control invariant set for the system (1) subject to the constraints (2), if

$$
x(t) \in \mathcal{C} \Rightarrow \exists u(t) \in \mathcal{U}: f(x(t), u(t)) \in \mathcal{C}, \forall t \in \mathbb{N}^{+}
$$

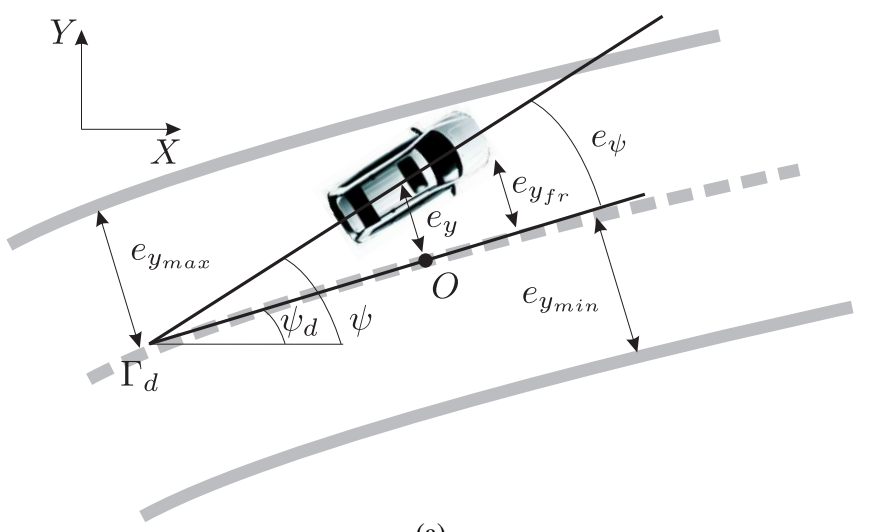

(a)

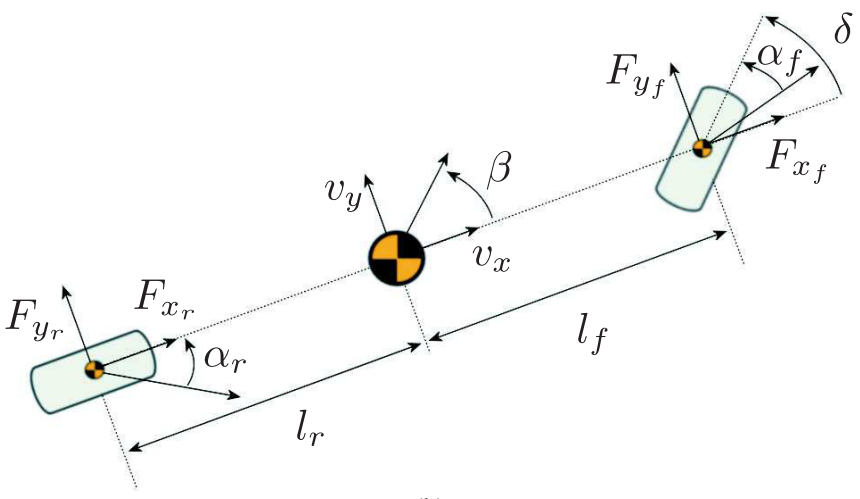

(b)

Fig. 2. Vehicle modeling notation.

Definition 2 (Maximal Control Invariant Set $\mathcal{C}_{\infty}$ ): The set $\mathcal{C}_{\infty}$ is said to be the maximal control invariant set for the system (1) subject to the constraints (2), if it is control invariant and contains all control invariant sets contained in $\mathcal{X}$.

For all states contained in the maximal control invariant set $\mathcal{C}_{\infty}$ there exists a control law, such that the system constraints are never violated.

\section{Modeling}

In this section, we present the vehicle mathematical model used in Section V, as basis of the threat assessment algorithm.

\section{A. Vehicle Modeling}

Consider the vehicle model sketched in Figure 2. The vehicle motion within the lane, subject to the lateral and yaw dynamics, is described by the following set of differential equations

$$
\begin{aligned}
m \dot{v}_{y} & =-m v_{x} \dot{\psi}+2\left[F_{y_{f}}+F_{y_{r}}\right], \\
J_{z} \ddot{\psi} & =2\left[l_{f} F_{y_{f}}-l_{r} F_{y_{r}}\right], \\
\dot{e}_{\psi} & =\dot{\psi}-\dot{\psi}_{d}, \\
\dot{e}_{y} & =v_{y}+v_{x} e_{\psi}, \\
\dot{\delta} & =u,
\end{aligned}
$$

where $m$ and $J_{z}$ denote the vehicle mass and yaw inertia, respectively, $l_{f}$ and $l_{r}$ are the distances of the vehicle center 
of gravity from the front and rear axles, respectively, $v_{x}$ and $v_{y}$ are the longitudinal and lateral velocities, respectively, in the vehicle body frame, $\dot{\psi}$ is the turning rate, where $\psi$ denotes the vehicle orientation w.r.t. the fixed global frame $(X, Y)$ in Figure 2(a). $F_{y_{f}}, F_{y_{r}}$ are the lateral tire forces at the front and rear axles, respectively. In (4c) and (4d), $e_{\psi}$ and $e_{y}$ denote the vehicle orientation and position errors, respectively, w.r.t. the road centerline and $\psi_{d}$ is the desired vehicle orientation, i.e., the slope of the tangent to the curve $\Gamma_{d}$ in the point $O . \delta$ denotes the front steering angle as depicted in Figure 2(b) and $u$ is the external input signal. The choice of input signal is commented next, in Section IV-B.

The lateral tire forces in (4a) and (4b) are generated at the tire contact patch and are, in general, nonlinear functions of the vehicle states. Accurate physical modeling of tire forces is quite involving and several models have been proposed in the literature over the past two decades. An exhaustive review of existing tire models can be found in [12]. In this paper, we compute the lateral tire forces as

$$
F_{y, i}=-C_{i} \alpha_{i}, i \in\{f, r\},
$$

where $C_{i}$ are the tire cornering stiffness coefficients at the two axles and $\alpha_{i}$ are the tire slip angles which, for small values, can be approximated as

$$
\begin{aligned}
& \alpha_{f}=\frac{v_{y}+l_{f} \dot{\psi}}{v_{x}}-\delta, \\
& \alpha_{r}=\frac{v_{y}-l_{r} \dot{\psi}}{v_{x}} .
\end{aligned}
$$

Remark 1: The simplified linear tire model (5) well approximates more complex nonlinear tire characteristics [13] for small tire slip angles, i.e., $\alpha_{i} \in\left[\alpha_{i_{\min }}, \alpha_{i_{\max }}\right]$. However, this interval also corresponds to a "normal driving" region where drivers usually operate [14], [15].

\section{B. System Constraints}

In Figure 3, an illustration of two different scenarios, targeted by the threat assessment method presented in this paper, is provided. In Figure 3(a) the roadway departure occurs because of a vehicle drift, while in Figure 3(b) the vehicle instead operates beyond its stability limits.

Next we define a set of operating conditions, in the space of the states and inputs of system (4), corresponding to stable driving within the lane boundaries and the system physical limitations. In order to avoid possible vehicle instability due to the effects of the tire nonlinearities (see Remark 1), the vehicle can be forced to operate in a region of the state space corresponding to limited values of the tire slip angles $\alpha$

$$
\alpha_{i_{\min }} \leq \alpha_{i} \leq \alpha_{i_{\max }}, i \in\{f, r\}
$$

Furthermore, constraints on vehicle position are set by the limited lane width. In order to formulate the constraints on the vehicle position within the lane, we denote by $e_{y_{i j}}, i \in$ $\{f, r\}, j \in\{l, r\}$, the distances of the four vehicle corners

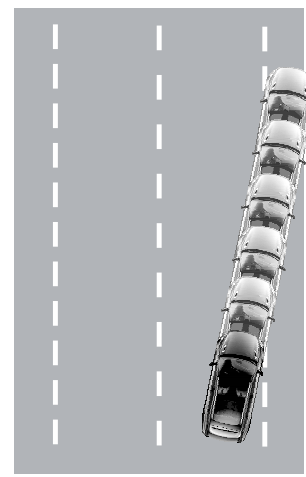

(a)

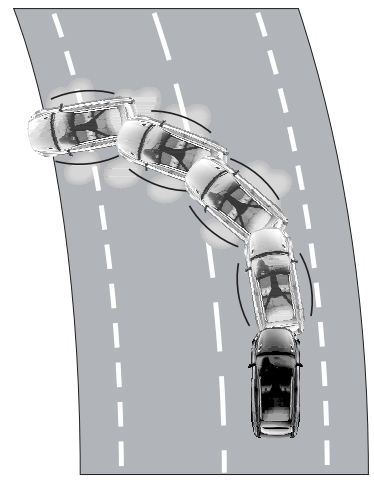

(b)
Fig. 3. Two pre-crash scenarios that might lead to unwanted roadway departures. In (a) the vehicle leaves the road while traveling on a straight, possibly due to drowsiness or distraction, while in (b) the vehicle loses control while negotiating a curve. The latter might typically occur due to excessive speed.

from the lane centerline. By assuming small orientation errors,

$$
\begin{array}{ll}
e_{y_{f l}}=e_{y}+\frac{c}{2}+a e_{\psi}, & e_{y_{f r}}=e_{y}-\frac{c}{2}+a e_{\psi}, \\
e_{y_{r l}}=e_{y}+\frac{c}{2}-b e_{\psi}, & e_{y_{r r}}=e_{y}-\frac{c}{2}-b e_{\psi},
\end{array}
$$

where $c$ is the vehicle width, $a$ and $b$ are the distances of the center of gravity from the front and rear vehicle bumpers, respectively. The constraints on the vehicle position and slip angles then read

$$
\begin{aligned}
& e_{y_{\min }} \leq e_{y_{i j}} \leq e_{y_{\max }}, \\
& \alpha_{i_{\min }} \leq \alpha_{i} \leq \alpha_{i_{\max }}, i \in\{f, r\}, j \in\{l, r\},
\end{aligned}
$$

where $e_{y_{\min }}$ and $e_{y_{\max }}$ set the maximum allowed deviation from the lane centerline.

Remark 2: The model (4)-(6), subject to constraints (9), describes the stable vehicle motion within the lane. In such operating conditions the small orientation errors assumption is deemed reasonable.

Constraints on the steering angle $\delta$ are set by mechanical limitations while the steering rate $\dot{\delta}$ is constrained by the driver's ability to change the steering angle. Hence, we consider the steering rate $\dot{\delta}$ as input signal and impose the following constraints,

$$
\begin{gathered}
-\delta_{\max } \leq \delta \leq \delta_{\max }, \\
-\frac{\dot{\delta}_{\max }^{s w}}{g} \leq u \leq \frac{\dot{\delta}_{\max }^{s w}}{g},
\end{gathered}
$$

where $\dot{\delta}_{\text {max }}^{s w}$ denotes the maximally attainable steering angle rate at the steering wheel and $g$ is the gear ratio between the steering wheel and the tires.

The model (4)-(6), along with the constraints (9), (10) can be compactly written as

$$
\dot{x}(t)=A x(t)+B u(t)+E w(t)
$$

subj. to

$$
\left[\begin{array}{cc}
H_{x} & 0 \\
0 & H_{u}
\end{array}\right]\left(\begin{array}{l}
x \\
u
\end{array}\right) \leq\left[\begin{array}{l}
h_{x} \\
h_{u}
\end{array}\right]
$$


where $x=\left[v_{y}, \dot{\psi}, e_{\psi}, e_{y}, \delta\right]^{T}, u=\dot{\delta}$ and $w=\dot{\psi}_{d}$ are the state, the input and the disturbance vectors, respectively. The definition of matrices $A, B, E, H_{x}, H_{u}, h_{x}$ and $h_{u}$ is straightforward and omitted due to lack of space.

\section{SET BASED THREAT ASSESSMEnT}

In this section we propose a threat assessment method based on the vehicle model presented in Section IV.The idea underlying the proposed method is to first introduce a set of constraints that the vehicle state and input trajectories have to satisfy in order to "drive safely". The constraints are set by the lane boundaries and the stability limits the vehicle should not exceed, i.e., the inequality (11b). Constraints satisfaction implies that the vehicle is traveling within the lane boundaries in a stable operating region. The threat assessment problem is then reformulated as a constraints satisfaction problem over a future time horizon, solved through the predictive model based method described next.

Based on the same underlying ideas, a threat assessment algorithm is presented in [16]. In [16], the steering angle $\delta$ is computed through a driver model which is restricted to the class of linear state feedback control laws. In this paper, instead, we remove this restriction and base the threat assessment on the existence of any steering trajectory subject to constraints (10) and guaranteeing a stable vehicle motion within the lane boundaries. As a consequence of this relaxation, the threat assessment algorithm presented in this paper is less restrictive than in [16].

Based on the vehicle model, every time step we calculate a "safe set". This is the set of vehicle states at current time $t$ for which a steering trajectory exists such that, over a finite time horizon, the vehicle state evolves to a given final set without violating constraints (11b). The safe set is updated in receding horizon, as new information about the surrounding environment are available, e.g., the coming road curvature in our lane guidance application. Once the safe set is computed, a set membership test on the current vehicle state is performed. If the current state belongs to the safe set, a steering trajectory exists such that the vehicle can be driven over the considered future time horizon without exceeding its stability limits and leaving the lane.

We introduce the following assumptions on the disturbance signal $w$ (i.e., the road curvature)

Assumption 1: $w(t) \in \mathcal{W}, \forall t \geq 0$, where $\mathcal{W} \subseteq \mathbb{R}^{2}$ is a polyhedron that contains the origin in its interior.

Assumption 2: Every time instant $t$, the disturbance $w(t)$ is known over a finite time horizon of $N$ steps.

Remark 3: The sensing technologies used in e.g. [17], [18], [19] can be used in order to obtain the road geometry information over a finite time horizon.

We discretize the model (11a) with a sampling time $T_{s}$, to obtain the discrete time constrained system with disturbances

$$
\begin{aligned}
& x(t+1)=A^{d} x(t)+B^{d} u(t)+E^{d} w(t) \\
& \text { subj. to } \\
& {\left[\begin{array}{cc}
H_{x} & 0 \\
0 & H_{u}
\end{array}\right]\left(\begin{array}{l}
x \\
u
\end{array}\right) \leq\left[\begin{array}{l}
h_{x} \\
h_{u}
\end{array}\right]}
\end{aligned}
$$

where, for the sake of simple notation, we have denoted the state, the input, the disturbance and the time index with the same symbols as in the continuous time model (11).

Denote by $\mathcal{X}_{\text {feas }}$ the set of admissible states

$$
\mathcal{X}_{\text {feas }}=\left\{x \in \mathbb{R}^{5}: H_{x} x \leq h_{x}\right\} .
$$

Every time instant, we consider a terminal target set $\mathcal{T} \subseteq$ $\mathcal{X}_{\text {feas }}$. Further details about the choice of $\mathcal{T}$ are provided next in Section V-A. Moreover, denote by $W_{t}=$ $\left[w_{t}, w_{t+1}, \ldots, w_{t+N-1}\right]$, the sequence of disturbance samples over the time horizon $[t, t+N-1]$ and by $W_{t, i}=$ $\left[w_{t+i}, \ldots, w_{t+N-1}\right]$ any sequence extracted from $W_{t}$. We compute the sequence of states sets $X_{t}\left(W_{t}\right)=$ $\left[\mathcal{X}_{t}, \mathcal{X}_{t+1}, \ldots, \mathcal{X}_{t+N-1}\right]$ as:

$$
\begin{array}{rr}
\mathcal{X}_{t+i}\left(W_{t, i}\right) & =\mathcal{X}_{\text {feas }} \bigcap \operatorname{Pre}_{f}\left(\mathcal{X}_{t+i+1}, w_{t+i}\right), \\
& i=N-1, \ldots, 0, \\
\mathcal{X}_{t+N} & =\mathcal{T},
\end{array}
$$

where, $f$ denotes the right hand side of the (12a). We call the set $\mathcal{X}_{t}$ the safe set at time $t$.

We observe that the calculation of the sequence $X_{t}\left(W_{t}\right)$ is performed every time step, based on the updated disturbance sequence $W_{t}$. Moreover, in (14), with a slight abuse of notation, as second argument a vector is used instead of a set in the set operator Pre. $(\cdot, \cdot)$.

In summary, the proposed threat assessment algorithm is made of three main steps to be performed every time step

1) select the terminal target set $\mathcal{T}$,

2) based on the future disturbance sequence $W_{t}$ and the set $\mathcal{T}$, perform the backward calculation of the sequence of safe sets $\mathcal{X}_{t+i}$ according to (14),

3 ) check whether the current state $x(t)$ belongs to the safe set $\mathcal{X}_{t}$, in order to assess the driver's ability in safely driving the vehicle from the current state to the target set $\mathcal{T}$ over the future horizon of $N$ steps.

The steps of the method are detailed next in Algorithm 5.1.

Remark 4: By construction, if the state of the system (12) at the current time $t$ belongs to the safe set $\mathcal{X}_{t}$ (Step 10 of Algorithm 5.1), a steering trajectory exists such that the vehicle can be driven over the next $N$ time steps, while operating within its stability limits and without leaving the lane. That is, over the future $N$ time steps, the vehicle can be driven within the lane boundaries, while operating in a region of the system states and inputs space where the driver is deemed capable of driving without loosing vehicle stability (see Remark 1).

Remark 5: The advantage of formulating the threat assessment problem as a constraint satisfaction problem is the existence of several efficient methods for solving such problems [20]. An advantage of Algorithm 5.1 is that it provides a polyhedral representation of safe sets rather than just a boolean answer. As pointed out in Section I, a polyhedral representation of the safe set can be exploited for both control and verification purposes.

As last remark of this section, we observe that the Algorithm 5.1 checks the existence of a steering trajectory capable 


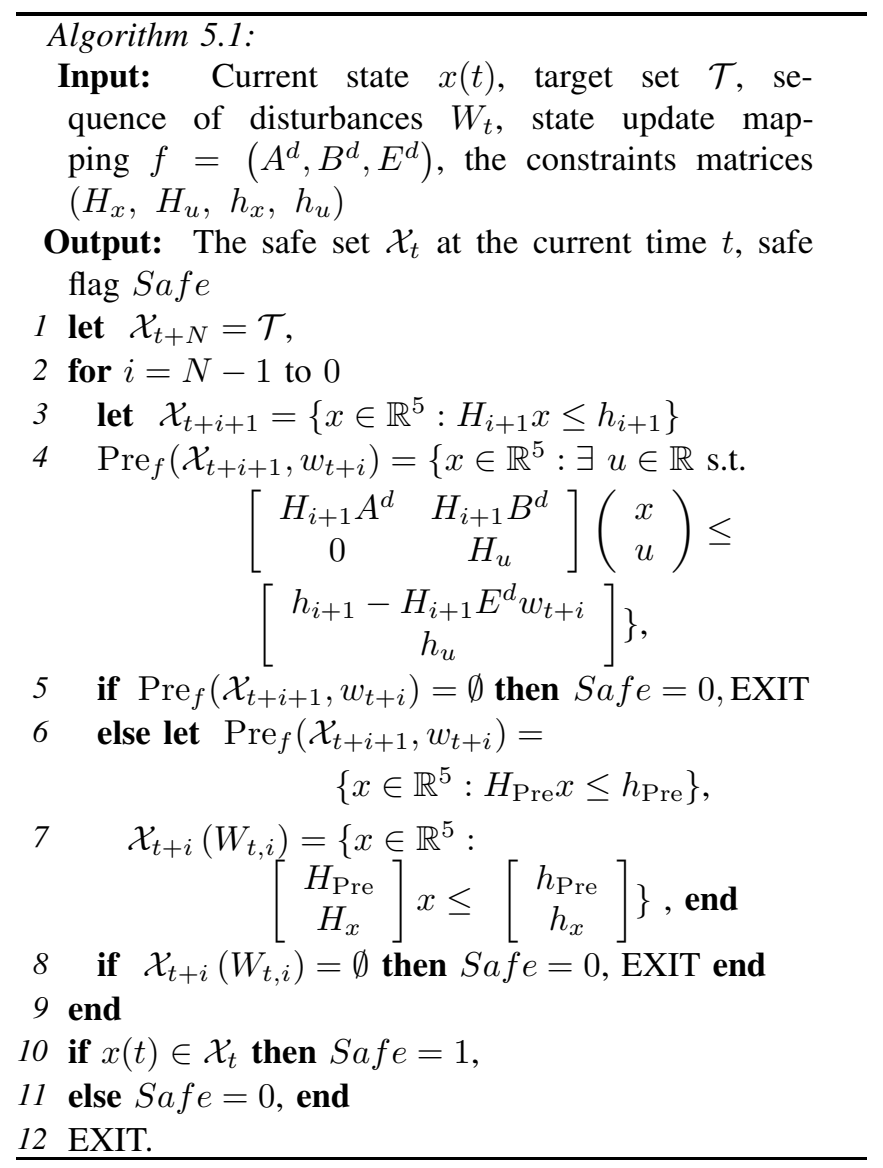

of driving the vehicle, modeled by (11a), from the current state $x(t)$ to the target set $\mathcal{T}$. Hence, such trajectory is not guaranteed to exist after the time $t+N$. In the next section we comment the choice of the target set $\mathcal{T}$ and propose a method for guaranteeing persistent constraint satisfaction, i.e., that the driver will be capable of maintaining the vehicle within $\mathcal{T}$, for $t>t+N$.

\section{A. Terminal Set}

The choice of the terminal set $\mathcal{T}$ in the threat assessment Algorithm 5.1 affects the effectiveness and the conservativeness of the algorithm. Indeed, the simplest choice is setting $\mathcal{T}=\mathcal{X}_{\text {feas }}$. In this case, Algorithm 5.1 can be used to assess the driver's ability of safely driving only over the future $N$ time steps. As alternative, the set $\mathcal{T}$ could be chosen as $\mathcal{T}=\mathcal{C}_{\infty}$, where $\mathcal{C}_{\infty} \subseteq \mathcal{X}_{\text {feas }}$ is the maximal robust control invariant set for the system with inputs (12a) subject to the constraints $(12 b)$. We recall that in this case,

$x(t+N) \in \mathcal{C}_{\infty} \Rightarrow x(t+N+k) \in \mathcal{C}_{\infty}, \forall w(t) \in \mathcal{W}, k \in \mathbb{N}^{+}$,

that is, the vehicle will be kept within the lane and its stability limits, despite all admissible lane curvature beyond the lookahead point. Nevertheless, setting the final set equal to the maximal robust positive invariant set might lead to high conservativeness of the threat assessment algorithm.

\section{RESUlTS}

Experiments on a test track have been performed in order to validate the threat assessment method described in Section V. The test vehicle has been driven several laps with different test drivers. The drivers adopted different driving styles, ranging from normal driving to rough driving. The test vehicle was equipped with a differential GPS, with built in Inertial Measurement Unit (IMU) and a digital map of the test track. Through this sensors set, estimates of the full state and disturbance vectors were available (see Remark 3 for alternative sensor setups).

In Figure 4(a) we illustrate the scenario considered next for experimental validation. In the considered scenario, the driver is negotiating a curve at a constant speed of approximately $90 \mathrm{~km} / \mathrm{h}$. Consider the vehicle positions 1 and 2 on the track, shown in figure 4(a). Denote by $t_{1}$ and $t_{2}$ the time instants, when the vehicle is in positions 1 and 2 , respectively. The dashed lines starting from the vehicle denote the horizon of $N$ steps, over which the disturbance is assumed to be known. In the two vehicle positions we have computed the safe sets $\mathcal{X}_{t_{1}}$ and $\mathcal{X}_{t_{2}}$, respectively, according to the threat assessment algorithm in Section V, where we have set $\mathcal{T}=\mathcal{X}_{\text {feas }}$ as terminal set and used the following parameters

$$
\begin{aligned}
& \alpha_{f_{\max }}=\alpha_{r_{\max }}=-\alpha_{f_{\min }}=-\alpha_{r_{\min }}=4^{\circ}, \\
& \delta_{\max }=10^{\circ}, \dot{\delta}_{\max }^{s w}=300^{\circ} / \mathrm{s}, N=35, T_{s}=0.01 \mathrm{~s}, \\
& e_{y_{\max }}=-e_{y_{\min }}=1.56 \mathrm{~m} .
\end{aligned}
$$

In Figures 4(b) and 4(c) the polyhedra $\mathcal{X}_{t_{1}}^{3,4}$ and $\mathcal{X}_{t_{2}}^{3,4}$ are shown, where

$\mathcal{X}_{t}^{3,4}=\mathcal{X}_{t} \bigcap\left\{x \in \mathbb{R}^{5}:\left[\begin{array}{lllll}1 & 0 & 0 & 0 & 0 \\ 0 & 1 & 0 & 0 & 0 \\ 0 & 0 & 0 & 0 & 1\end{array}\right] x=\left[\begin{array}{l}x^{1}(t) \\ x^{2}(t) \\ x^{5}(t)\end{array}\right]\right\}$

and the superscript $i$ in $x^{i}(t)$ denote the $i$-th component of the current vehicle state vector $x(t)$. The states $x\left(t_{1}\right)$ and $x\left(t_{2}\right)$ are marked with a circle in Figures $4(\mathrm{~b})$ and $4(\mathrm{c})$, respectively. We observe that $x\left(t_{1}\right) \in \mathcal{X}_{t_{1}}^{3,4}$ and $x\left(t_{2}\right) \notin$ $\mathcal{X}_{t_{2}}^{3,4}$. Hence, according to the proposed threat assessment algorithm, from the initial state $x\left(t_{1}\right)$, it is possible to safely drive the vehicle over a horizon of $N$ steps without violating any of the state and input constraints. From the initial state $x\left(t_{2}\right)$, on the other hand, the vehicle state trajectory is predicted to violate the constraints (13). The measured state trajectories are also reported in Figures 4(b) and (c) with dashed lines. We observe that in Figure 4(b), starting from the initial state $x\left(t_{1}\right)$, the state trajectory entirely evolves within the set $\mathcal{T}_{1}=\bigcup_{t=t_{1}}^{t_{1}+N-1} \mathcal{T}^{3,4}$, where the sets $\mathcal{T}^{3,4}$ are obtained by replacing $\mathcal{X}_{t}$ with $\mathcal{T}$ in (15). In Figure 4(c), instead, we observe that the state trajectory leaves the set $\mathcal{T}_{2}=\bigcup_{t=t_{2}}^{t_{2}+N-1} \mathcal{T}^{3,4}$, i.e., the vehicle violates the bounds on the lateral deviation from the lane centerline. 


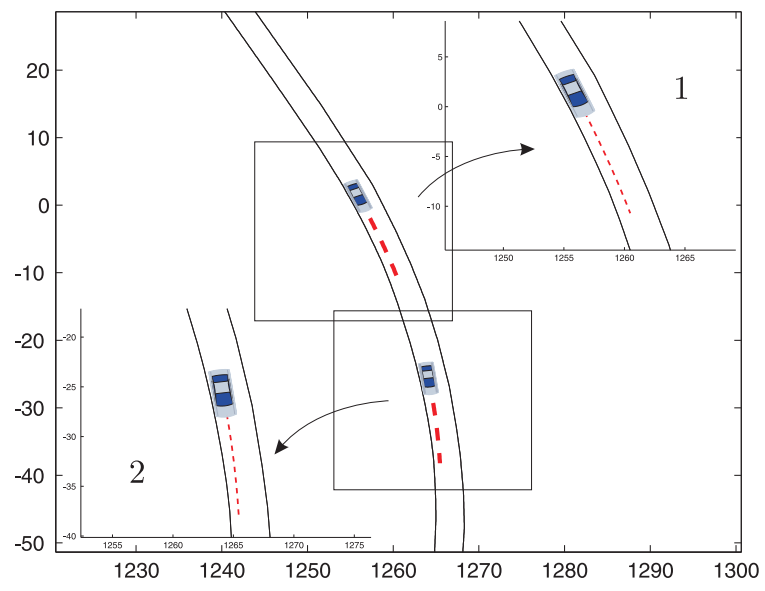

(a)

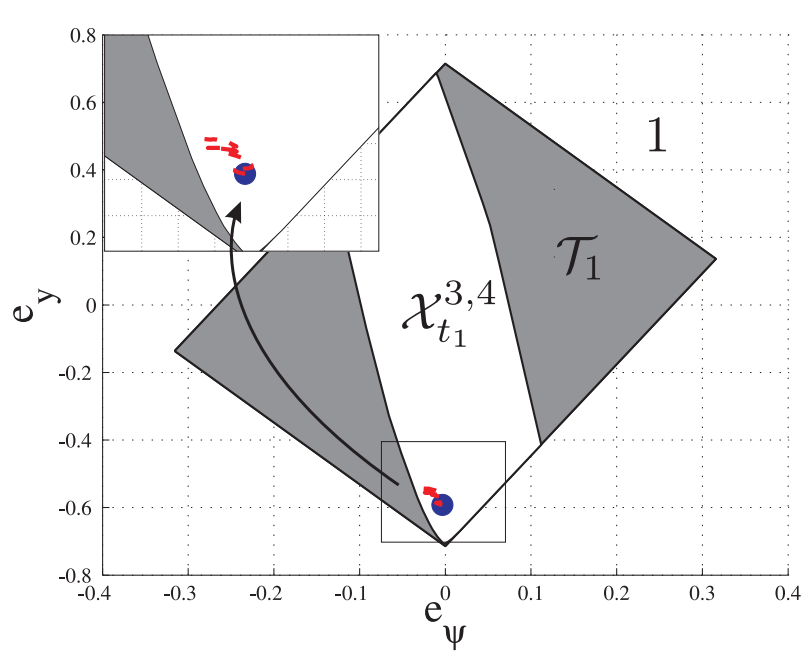

(b)

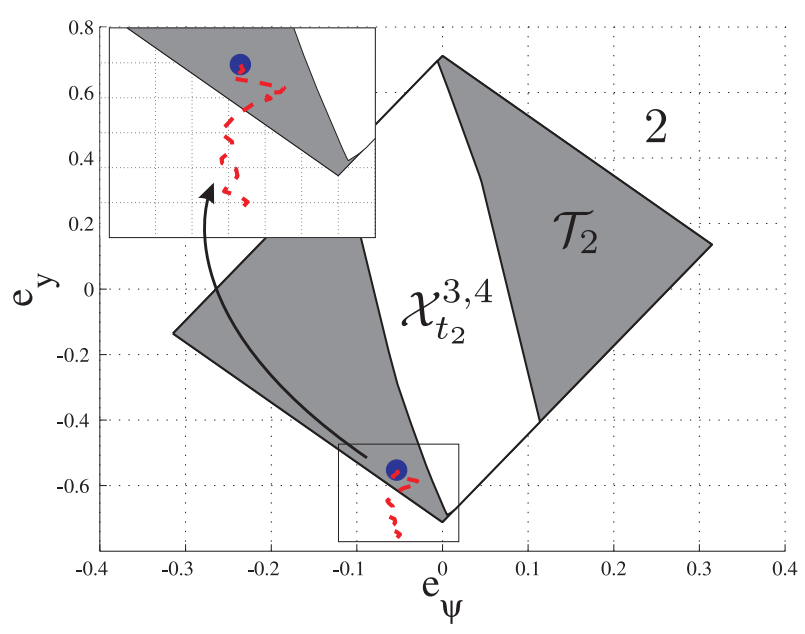

(c)

Fig. 4. Plot (a) shows the vehicle while negotiating a curve. Plot (b) shows the safe set at point 1 in (a) while plot (c) shows the safe set at point 2 . In plots (b) and (c), the circles denote the current state while the dashed line shows the measured state trajectory over the horizon.

\section{CONCLUSION AND FUTURE WORKS}

We have presented a model based threat assessment method for semi-autonomous vehicles. The algorithm is based on reachability analysis tools and set invariance theory. The method has been validated offline using experimental data. The obtained results demonstrate that the proposed method can effectively predict lane crossing and vehicle instability over a future, finite time horizon, allowing the activation of an autonomous intervention.

The preliminary results presented in this manuscript motivate further investigations aiming at analyzing the impact of measurements errors and model uncertainties on the performance of the proposed threat assessment method and proposing approaches in order to compensate for them.

\section{REFERENCES}

[1] M. Althoff, O. Stursberg, and M. Buss, "Model-based probabilistic collision detection in autonomous driving," IEEE Transactions on Intelligent Transportation Systems, vol. 10, no. 2, pp. 199-310, June 2009.

[2] A. M. Bayen, I. M. Mitchell, and C. J. Tomlin, "Aircraft autolander safety analysis through optimal control-based reach set computation," Journal of Guidance, Control and Dynamics, vol. 30, no. 1, pp. 68-77, January-February 2007.

[3] I. M. Mitchell, A. M. Bayen, and C. J. Tomlin, "A time-dependent hamilton-jacobi formulation of reachable sets for continuous dynamic games," IEEE Transactions on Automatic Control, vol. 50, no. 7, pp. 947-957, July 2005.

[4] M. Ali, P. Falcone, and J. Sjöberg, "A Predictive Approach To Roadway Departure Prevention," In Proc. 21st International symposium on dynamics of vehicles on roads and tracks, 2009.

[5] P. Grieder, "Efficient Computation of Feedback Controllers for Constrained Systems," Ph.D. dissertation, 2004.

[6] S. V. Rakovic, "Robust control of constrained discrete time systems: Characterization and implementation," Ph.D. dissertation, Imperial College London, London, United Kingdom, July 2005.

[7] E. C. Kerrigan, "Robust constraints satisfaction: Invariant sets and predictive control," Ph.D. dissertation, Department of Engineering, University of Cambridge, Cambridge, England, 2000.

[8] F. Blanchini, "Set invariance in control - a survey," vol. 35, no. 11, pp. 1747-1768, Nov. 1999

[9] D. P. Bertsekas and I. B. Rhodes, "On the minimax reachability of target sets and target tubes," Automatica, vol. 7, pp. 233-247, 1971.

[10] D. P. Bertsekas, "Control of uncertain systems with a set-membership description of the uncertainty," Ph.D. dissertation, MIT, 1971.

[11] I. Kolmanovsky and E. G. Gilbert, "Theory and computation of disturbance invariant sets for discrete-time linear systems," Mathematical Problems in Egineering, vol. 4, pp. 317-367, 1998.

[12] J. Svedenius, "Tire modeling and friction estimation," $\mathrm{Ph} . \mathrm{D}$. dissertation, Department of Automatic Control, Lund University, Lund, Sweden, April 2007.

[13] E. Bakker, L. Nyborg, and H. Pacejka, "Tyre Modeling for Use in Vehicle Dynamics Studies," SAE, vol. Paper 870421, 1989.

[14] R. Rajamani, Vehicle Dynamics and Control. Springer, 2006.

[15] U. Kiencke and L. Nielsen, Automotive Control Systems. Springer, 2005.

[16] P. Falcone, M. Ali, and J. Sjöberg, "Set-Based Threat Assessment in Lane Guidance Applications," IFAC Symposium Advances in Automotive Control, 2010.

[17] A. Eidehall, J. Pohl, and F. Gustafsson, "Joint road geometry estimation and vehicle tracking," Control Engineering Practice, 2007.

[18] J. Jansson, "Collision avoidance theory with application to automotive collision mitigation," Ph.D. dissertation, 2005.

[19] M. Bertozzi, A. Broggi, and A. Fascioli, "Vision-based intelligent vehicles: State of the art and perspectives," Robotics and Autonomous Systems, vol. 32, no. 1, pp. 1-16, juli 2000. [Online]. Available: http://linkinghub.elsevier.com/retrieve/pii/S0921889099001256

[20] S. Boyd and L. Vandenberghe, Convex optimization. Cambridge Univ Pr, 2004. 